\title{
Unnecessary Overtreatment Index: a useful tool in family practice
}

Índice de Sobretratamento Desnecessário: uma ferramenta útil para a medicina de família

Índice de Sobretratamiento Innecesario: una herramienta útil para la medicina familiar

Mohammad Zakaria Pezeshki M.D. Department of Community Medicine, Tabriz Medical School, Tabriz, Iran. zakaria.pezeshki@gmail.com

(Corresponding author)

Sina Pezeshki, M.D. Department of Community Medicine, Tabriz Medical School, Tabriz, Iran. sina.pezeshki@gmail.com

One of the most important aspects of quaternary prevention is avoiding the exposure of the patients to unnecessary overtreatment. In this commentary we explain how a valid and reliable double blinded randomized controlled clinical trial (RCT) with a good external validity may help a physician to estimate the magnitude of unnecessary overtreatment.

\section{Unnecessary Overtreatment Index}

Based on RCTs, two groups of patients may receive unnecessary overtreatments. The first group consists of patients who will not respond to medication or surgery. The second group consists of patients who respond to placebo interventions (either a medication or a sham surgery). Here we define an index to cover these two groups: Unnecessary Overtreatment Index (UOI). RCTs compare a medication with placebo intervention. Based on the finding of RCTs, the UOI is defined as summation of two proportions: the proportion of patients who do not respond to medication/surgery and the proportion of patients who are improved by the placebo intervention as well.

\section{Calculating the UOI}

Assuming that a RCT has a very good external validity, a physician wants to use its findings in his practice for treating his patients. Moreover, the RCT shows that 30/100 of patients who received medication/surgery have improved and for the placebo intervention group only 20/100 patients have also improved. In this example, UOI is summation of (1-30/100) and 20/100. It means if a physician prescribes the medication/surgery to 100 patients then 90 of these patients will be treated unnecessarily. Seventy out of 90 are patients who will not be improved and 20 out of 90 are patients who would be improved even if they received placebo intervention. The 95\% Confidence Interval can be calculated for UOI, if necessary.
Keywords:

Indexes

Evaluation of the Efficacy-

Effectiveness of Interventions

Statistical Analysis

Medicalization

Quaternary Prevention

Palavras-chave:

Índices

Avaliação de Eficácia-Efetividade

de Intervenções

Análise Estatística

Medicalização

Prevenção Quaternária

Palabras clave:

Índices

Evaluación de Eficacia-Efectividad de Intervenciones

Análisis Estadístico Medicalización

Prevención Cuaternaria 


\section{Relationship between UOI and Efficacy}

An interesting point is the relation of UOI with Efficacy of medication/surgery. Efficacy in a RCT is defined as the difference between improvement in the medication/surgery group and the placebo intervention control group. In the previous example, the efficacy consists of 10/100 (30/100 minus 20/100). As efficacy shows the proportion of patients that are treated necessarily and the UOI shows the proportion of the patients that are treated unnecessarily, then it is clear that efficacy could be defined as "1-UOI". Then, it can be stated that UOI=1-Efficacy. When in a RCT the improvement in medication/surgery arm is equal to the improvement in placebo intervention arm, then the efficacy is zero, and consequently, the UOI is $100 \%$. This means that if physicians do not take into account the findings of RCT and prescribe the RCT medication to all their patients, then all of them will be overtreated unnecessarily.

However, there are two remaining questions that need to be addressed: (1) what are the percentages of those patients who may be treated by placebo? And (2) what are the percentages of those patients who will not respond either to medication or placebo interventions? By determining the two components of UOI it is possible to answer these questions. Unfortunately, in the majority of the cases the efficacy of drug in a RCT is small, thus usually the UOI is very high. When the medication/surgery is recommended for a life-threatening condition, a large UOI is commonly acceptable for both physicians and patients. Nevertheless, when a medication is recommended for long duration, which offers partial improvements to mild chronic mental or physical conditions, a large UOI may not be acceptable either by physicians or patients. 